\title{
Molecular basis and promise of genetic therapy for diabetic dyslipidaemia: studies in an animal model of diabetes
}

\author{
L. Chan, K. Kobayashi \\ Departments of Cell Biology and Medicine, Baylor College of Medicine, Houston, Texas, USA
}

Atherosclerotic cardiovascular disease is a major complication of diabetes mellitus. It is the leading cause of morbidity and mortality in patients with non-insulin-dependent diabetes mellitus (NIDDM). Its prevalence among insulin-dependent diabetic patients (IDDM) has been increasing because of our success in reducing the early mortality of these patients through better medical care and treatment. Multiple factors predispose diabetic patients to premature cardiovascular complications. Diabetic dyslipidaemia is a major underlying risk factor that leads to accelerated atherosclerosis in such patients.

The dyslipidaemia of diabetes includes abnormalities in each of the major classes of plasma lipoproteins: very low density lipoproteins (VLDL), intermediate density lipoproteins (IDL) or VLDL remnants, and high density lipoproteins (HDL). Hypertriglyceridaemia is very common among IDDM and NIDDM patients. It is associated with elevated plasma VLDL and VLDL remnants. The VLDL are usually large triglyceride-rich particles. VLDL remnants, on the other hand, are dense and cholesterolenriched particles. Both increased production and reduced clearance are involved in the VLDL and remnant abnormalities. LDL levels appear to be elevated more often in NIDDM patients than in nondiabetic individuals. The LDL particles in diabetic patients tend to be dense and small. In contrast, HDL particles in NIDDM are usually larger and more triglyceride-rich than those in non-diabetic subjects.

Corresponding author: Dr. L. Chan, Departments of Cell Biology and Medicine, Baylor College of Medicine, Houston, Texas 77030, USA

A bbreviations: NIDDM, Non-insulin-dependent diabetes mellitus; IDDM, insulin-dependent diabetes mellitus; VLDL, very low density lipoproteins; IDL, intermediate density lipoproteins; HDL, high density lipoproteins; LPL, lipoprotein lipase.
The mechanism by which diabetic dyslipidaemia exerts its atherogenic effects is complex and incompletely understood. We have examined the $d b / d b$ mouse to see whether it is an appropriate model in which to study the pathogenesis and treatment of diabetic dyslipidaemia.

The C57BL/KsJ-db/db mice (abbreviated $\mathrm{db} / \mathrm{db}$ mice) were described 30 years ago [1]. They are homozygous for the "diabetes" ( $\mathrm{db})$ gene. Recently, the $d b$ gene was found to be the leptin receptor gene [2] and $\mathrm{db} / \mathrm{db}$ animals were found to have an mRNA splice donor site mutation that results in the inactivation of its gene product, the leptin receptor [2, 3]. These animals do not respond to leptin, a satiety factor in mice. With a C57BL/KsJ background, they also develop a severe form of NIDDM.

Compared to control C57BL/KsJ mice, $\mathrm{db} / \mathrm{db}$ mice were found to have hyperphagia, accelerated weight gain, hyperglycaemia and hyperinsulinaemia, starting before 6 weeks of age. At around 15 weeks, the insulin levels start to fall. They also have elevated plasma cholesterol (about twice normal) and triglyceride (about $65 \%$ above normal). By fast protein liquid chromatography (FPLC) analysis, they display elevated VLDL, LDL and HDL. Similar abnormalities were also observed by sequential ultracentrifugal analysis. Using non-denaturating gradient gel fractionation, we found that $\mathrm{db} / \mathrm{db}$ mouse LDL are of smaller size whereas their HDL tend to be larger than non-diabetic controls. In summary, $d b / d b$ mice display many of the lipoprotein abnormalities observed in humans: elevated VLDL, small dense LDL and triglyceride-rich large HDL. The one major difference, elevated plasma HDL, is probably the result of the absence of cholesteryl ester transfer protein in mice.

The similar lipoprotein abnormalities observed in the $d b / d b$ mouse suggest that it is an appropriate model of diabetic dyslipidaemia in humans. As a 
small animal model, it lends itself to easy manipulation and will be useful for testing novel therapeutic interventions.

The standard steps in the treatment of diabetic dyslipidaemia are: (i) diet therapy, (ii) hypoglycaemic therapy, and (iii) lipid-lowering drugs. These forms of treatment have had varying degrees of success in NIDDM patients; we often encounter diabetic patients who do not show an adequate response to such standard treatment modalities even when they are compliant. When somatic gene therapy becomes a safe and effective mode of treatment for dyslipidaemia in general, it is reasonable to consider extending its use in diabetic patients.

There is active research in the use of somatic gene therapy for treatment of non-diabetic dyslipidaemia. For example, LDL receptor gene transfer has been used as an experimental treatment for patients with LDL receptor deficiency [4]. In addition, a number of other therapeutic genes have been tested in animal models of dyslipidaemia (for review see $[5,6]$ ).

We believe that the following genes are potentially effective in reversing the dyslipidaemia of diabetes: (i) the LDL receptor, (ii) lipoprotein lipase (LPL) and (iii) the VLDL receptor. The lipid-lowering mechanism of the LDL receptor is well understood. It has proven efficacy in experimental animals in vivo [7-10] and appears to be safe in a few familial hypercholesterolaemic patients who received the LDL receptor transgene by an ex vivo approach [4]. LPL deficiency is common in diabetes. It is an underlying cause of the hypertriglyceridaemia and low HDL in diabetic patients. The use of LPL gene therapy appears to be a reasonable choice in patients with massive hypertriglyceridaemia who fail to respond to standard therapy. We have shown LPL gene transfer to be highly effective in eliminating VLDL in mice in vivo.

The VLDL receptor is a newly described member of the LDL receptor gene family. It is expressed in multiple tissues but is essentially undetectable in the liver. Its normal function is unknown. We have used adenovirus-mediated gene transfer to deliver the VLDL receptor transgene to the liver of LDL receptor-deficient mice [11]. The induced hepatic expression of the VLDL receptor was highly effective in reversing the hypercholesterolaemia in these animals. The ligand for the VLDL receptor appears to be apolipoprotein E-containing IDL. Since IDL is a precursor of LDL, both IDL and LDL were markedly lowered in response to VLDL receptor gene transfer.

We will test the efficacy of delivering the LDL receptor, LPL and VLDL receptor genes in reversing the diabetic dyslipidaemia of $\mathrm{db} / \mathrm{db}$ mice. New vectors and other transgene delivery methods are being developed by various laboratories. It is likely that a safe and efficient method for somatic gene therapy will be available in the near future. Our experiments in $\mathrm{db} / \mathrm{db}$ mice will be a step towards establishing whether these candidate therapeutic genes are safe and effective in experimental animals. We believe that a similar approach can eventually be applied to humans who suffer from diabetic dyslipidaemia that is resistant to the standards forms of treatment.

A cknowledgements. The work described in this paper was supported by a grant HL-16512 from the National Institutes of Health to L. Chan and a Fellowship from the Juvenile Diabetes Foundation International to K. Kobayashi. We thank Ms. I. A. Harrison for excellent secretarial assistance.

\section{References}

1. Hummel KP, Dickie MM, Coleman DL (1966) Diabetes, a new mutation in the mouse. Science 153: 1127-1128

2. Chen H, Charlat O, Tartaglia LA et al. (1996) Evidence that the diabetes gene encodes the leptin receptor: identification of a mutation in the leptin receptor gene in $\mathrm{db} / \mathrm{db}$ mice. Cell 84: 491-495

3. Lee GH, Proenca R, Montez JM et al. (1996) Abnormal splicing of the leptin receptor in diabetic mice. Nature 379: 632-635

4. Grossman M, Rader DJ, Muller DWM et al. (1995) A pilot study of ex-vivo gene therapy for homozygous familial hypercholesterolaemia. Nature Med 1: 1148-1154

5. Chan L (1995) Use of somatic gene transfer to study lipoprotein metabolism in experimental animals in vivo. Curr Opin Lipidol 6: 335-340

6. Gerard RD, Chan L (1996) Adenovirus-mediated gene transfer: strategies and applications in lipoprotein research. Curr Opin Lipidol 7: 105-111

7. Chowdhury JR, Grossman M, Gupta S, Chowdhury NR, Baker TR Jr, Wilson JM (1991) Long-term improvement of hypercholesterolemia after ex vivo gene therapy in LDLR-deficient rabbits. Science 254: 1802-1805

8. Ishibashi S, Brown MS, Goldstein JL, Gerard RD, Hammer RE, Herz J (1993) Hypercholesterolemia in LDL receptor knockout mice and its reversal by adenovirus-mediated gene delivery. J Clin Invest 92: 883-893

9. Kozarsky KF, McKinley DR, Austin LL, Raper DE, Stratford-Perricaudet LD, Wilson JM (1994) In-vivo correction of low density lipoprotein receptor deficiency in the Watanabe heritable hyperlipidemic rabbit with recombinant adenovirus. J Biol Chem 269: 13695-13702

10. Li J, Fang B, Eisensmith RC et al. (1995) In-vivo gene therapy for hyperlipidaemia: phenotype correction in Watanabe rabbits by hepatic delivery of the rabbit LDL receptor gene. J Clin Invest 95: 768-773

11. Kobayashi K, Oka K, Forte T et al. (1996) Reversal of hypercholesterolemia in low density lipoprotein receptor knockout mice by adenovirus-mediated gene transfer of the very low density lipoprotein receptor. J Biol Chem 271: 6851-6860 